\title{
Expert assistance
}

Endodontic cases can present a number of challenges, especially if a patient requires retreatment after a failed root canal procedure.

So, instead of stressing why don't you refer to EndoCare?

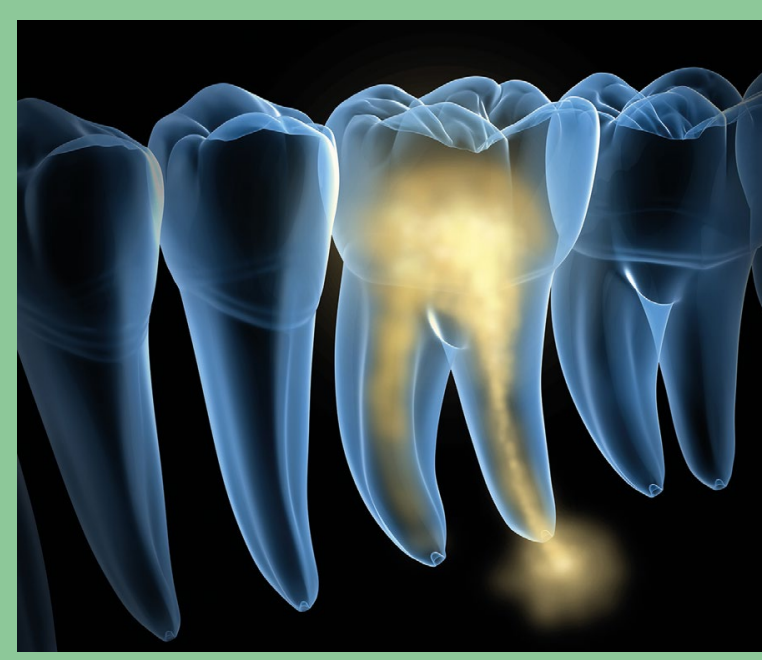

Our team has decades of experience in treating complex endodontic cases - in fact, we've saved tens of thousands of teeth that would have otherwise needed extraction. Plus, through the use of cutting-edge techniques, we can guarantee that treatment is as pain-free as possible.

We always return patients to your care with full details of any treatment performed, aftercare instructions and our suggested next steps.

To refer to us, you can either call one of our practices or fill out our patient referral form at https://endocare.v-forms.net/ refer-a-patient/

For further information call EndoCare on 02072240999 or visit www.endocare.co.uk.

\section{Think again}

If you have patients who don't believe orthodontics is right for them, with ClearCorrect, they can think again.

The ClearCorrect system works with every busy lifestyle. The aligners are discrete, nearly invisible and will fit comfortably over the teeth to gently guide them into position.

From the professional perspective, the aligners are part of a comprehensive digital workflow with the Straumann Group, utilising state-of the-art intraoral scanning that integrates seamlessly with the 3D digital set up for highly accurate treatment planning and smile simulations. Clinicians can even interact with the dental technicians fabricating the plan directly to ensure a customised approach for every patient.

This is a cuttingedge way to deliver quality orthodontics in your practice ClearCorrect can be fully integrated into a digital workflow, from treatment planning to monitoring.

With Clear Correct you will help patients achieve the smile of their dreams.

For more information on ClearCorrect visit www.straumann.com/clearcorrect/en/ home.html.



\section{A little something}

\section{from US...}

Here at Curaprox, we understand that kids need a little push when it comes to brushing their teeth - that's why we offer a bright and stimulating kids range to make oral health routines cheerier.

Our kids toothbrushes come in a range of exciting colours with soft, ultra-fine filaments to protect their gums. They can be paired with our delightful range of kids toothpastes which come in strawberry, watermelon and mint flavours - for the perfect oral health care routine.

Do you stock Curaprox in your practice? For those that do, from the 1st to the 31st of December, Curaprox is offering unmissable savings on kids toothpaste and toothbrushes, among other amazing special deals:

$\rightarrow$ When you buy 10 or more tubes of kids toothpaste, you can save $25 \%$ *

$\rightarrow$ When you buy 10 or more kids brushes, you can save $25 \%$ *

Consider recommending Curaprox as the ultimate product to help your younger patients find the joy in caring for their oral health.

For more information call 01480 862084, email info@curaprox.co.uk or visit www.curaprox.co.uk.

${ }^{*}$ Minimum order of $£ 100$ required to receive offer. Offers are available to residents of the United Kingdom and Northern Ireland only. Cannot be used in conjunction with any other offers.



